\title{
Pregabalin Administration Induces Alterations in Neural Tube Development During Early Embryonic Stages
}

\author{
Murat SAYIN ${ }^{1}$, Fatma Ela SIMSEK ${ }^{2}$ \\ ${ }^{1}$ Izmir Katip Celebi University, School of Medicine, Department of Neurosurgery, Izmir, Turkey \\ ${ }^{2}$ Izmir Katip Celebi University, School of Medicine, Department of Histology and Embryology, Izmir, Turkey
}

Corresponding author: Murat SAYIN smsayin@gmail.com

\section{ABSTRACT}

AIM: To evaluate the effect of pregabalin on neural tube development using early chick embryos.

MATERIAL and METHODS: Experiments were conducted on specific pathogen-free Leghorn chick embryos, which were equally divided into control and pregabalin-treated (therapeutic dose: $600 \mathrm{mg}$; supratherapeutic dose: $1200 \mathrm{mg}$ ) groups. The embryos were macroscopically and microscopically evaluated following pregabalin administration. Expression levels of mammalian target of rapamycin (mTOR), c-Jun N-terminal kinase (JNK), and microtubule-associated proteins 1A/1B light chain 3 (LC3) in the embryos were observed.

RESULTS: The embryos in the therapeutic dose group appeared more curved than those in the control group. The vesicles in the supratherapeutic dose group were more distinct but smaller than those in the control and therapeutic dose groups. mTOR expression was high in the control group and low in the therapeutic and supratherapeutic dose groups. JNK expression was low in the control and therapeutic dose groups and moderate in the supratherapeutic dose group. LC3 expression was moderate in the control and therapeutic dose groups and strong in the supratherapeutic dose group.

CONCLUSION: Pregabalin administration induced neural tube defects and fetal abnormalities in the chick embryos through increased autophagy due to enhanced apoptosis in the prenatal fetus.

KEYWORDS: Neural tube defects, Pregabalin, Antiepileptic drugs, Chick embryos

\section{INTRODUCTION}

$\mathrm{F}$ Tetal abnormalities, including neural tube defects, account for $65 \%$ of all malformations in newborns (4). The primary causes of neural tube defects include antiepileptic drug (AED) use by expecting mothers, malnutrition, toxicity, and radiation exposure (34). Antiepileptic drugs, such as valproic acid, phenobarbital, phenytoin, and carbamazepine, have been reported to induce neural tube defects in earlier studies (23); however, these studies lack reliable data, particularly regarding recently developed drugs. Other experiments conducted on drugs, such as oxcarbazepine, topiramate, and zonisamide, have reported fewer fetal malformations $(3,18$ 20,24,26,27,29,32).
Pregabalin, an AED, is the structural analog of the inhibitory neurotransmitter, $y$-amino butyric acid (GABA). Pregabalin regulates the activity of voltage gated $\mathrm{Ca}^{+2}$ channels by binding to their $a 2-\delta$ subunits, which has been reported to be the mechanism underlying the action of pregabalin in alleviating neuropathic pain, anxiety, and epileptic seizures (33). Pregabalin has been approved by the Food and Drug Administration (FDA) for neuropathic pain arising from postherpetic neuralgia, spinal cord injury, and diabetic peripheral neuropathy and also as adjunctive treatment for partial-onset seizures (30). Etemad et al. have reported limb and craniofacial anomalies in pregabalin induced rodent fetus (10). Furthermore, experiments performed in pregnant rabbits administrated with pregabalin have demonstrated low fetal weight and decreased ossification (6). 
To the best of our knowledge, there are no reports on the effects of pregabalin on the neural tube of developing chick embryos. Therefore, the present study was performed to investigate the effect of pregabalin on neural tube development in early chick embryos.

\section{MATERIAL and METHODS}

This study was conducted with the cooperation of the Histology Department Research Laboratory of Izmir Katip Celebi University Medical School. The experiments were performed on fertilized, specific pathogen-free Leghorn chick eggs obtained from the Republic of Turkey, Ministry of Agriculture and Rural Affairs, Bornova Veterinary Control and Research Institute.

\section{Incubation and Injection}

Experiments were performed on sixty eggs weighing $65 \pm 5 \mathrm{~g}$ (mean $\pm \mathrm{SD}$ ), incubated at $37.5 \pm 0.2^{\circ} \mathrm{C}$ and $60 \%-80 \%$ relative humidity for 24 hours. The eggs were repositioned on their axis every 2 hours. After 24 hours of incubation, all eggs that had reached the Hamburger-Hamilton stage 9 were opened (12), and observed under the $4 \times$ optical magnification. Then, the eggs were rinsed with $70 \%$ ethanol. The injection was given through a small hole. The drug was introduced under each embryonic disc using a 30-gauge syringe. After injection, the eggs were closed using sterile tape.

\section{Drug Preparation}

Pregabalin doses were estimated according to the weight of the eggs. An orally disintegrating $25-\mathrm{mg}$ capsule of pregabalin (Lyrica $^{\circledR}$ (pregabaline), NY, Pfizer Inc, 2018) was dissolved in sodium chloride solution. Subsequently, pregabalin solutions of two concentrations were prepared as the therapeutic (600 mg/70kg; $0.01 \mathrm{mg} / 0.01 \mathrm{ml})$ and supratherapeutic (1200 $\mathrm{mg} / 70 \mathrm{~kg}$; $0.02 \mathrm{mg} / 0.01 \mathrm{ml}$ ) dose and were injected under the embryonic discs using a 30 -gauge syringe.

\section{Groups}

The study design comprised three groups, each constituting 20 eggs. One group served as the control group, and the other two groups were treated with the therapeutic or supratherapeutic dose of pregabalin. In all the groups, the eggs were closed, sealed with a sterile tape following injection, and incubated for 72 hours. Then, the eggs were reopened, and the embryos were dissected using the waterfloating technique. Following the dissection, the embryos were immersed in a 10\% formalin solution for 24 hours. After 72 hours of incubation, the embryos were analyzed under a microscope using the Hamburger-Hamilton chick embryology classification system (12).

\section{H\&E and Immunohistochemistry}

All the samples were fixed in $10 \%$ formalin for 24 hours and embedded in paraffin. Five $\mu \mathrm{m}$-thick sections were cut and dewaxed overnight at $60^{\circ} \mathrm{C}$ and were then deparaffinized in xylene for 30 minutes. After ethanol dehydration, the sections were washed with distilled water and stained with hematoxylin and eosin (H\&E). Additional sections were also used for immunohistochemical staining. Tissues were first treated with $2 \%$ trypsin and incubated in $3 \% \mathrm{H}_{2} \mathrm{O}_{2}$ solution to inhibit endogenous peroxidase activity. They were then washed with PBS and incubated with anti-mTOR (sc-8319, Santa Cruz Biotechnology, Inc.), anti-JNK (sc-7345, Santa Cruz Biotechnology Inc., USA), and LC3 (LC3B, NB100-2220, Novus Biologicals Littleton, CO, USA) primary antibodies at 1:100 dilution with PBS for $18 \mathrm{~h}$ at $4^{\circ} \mathrm{C}$. After incubation with a secondary antibody, the sections were stained using a DAB-plus substrate kit (Thermofischer Scientific Waltham, MA, USA). To produce counterstaining, slides were then stained using Mayer's hematoxylin (72804E, Microm, Walldorf, Germany). Slides were subjected to blind assessment using a light microscope (Olympus BX-43, Tokyo, Japan). Negative controls received the same staining treatments and produced no labeling in any of the cases. Intensity of immunoreactivity for each slide was scored as mild (1), moderate (2), strong (3), or very strong (4).

\section{RESULTS}

Following 72 hours of incubation, the control chick embryos revealed clearly visible brain vesicles in the form of the telencephalon, diencephalon, mesencephalon, and metencephaIon. The initiation of pigmentation was observed on both the sides during the formation of the lens vesicle and the eye. The heart and the bulbus cordis were clearly located at their appropriate site. In addition, the descending aorta and the otic vesicle could be visibly distinguished among the structures. Additionally, the spinal cord was seen to have extended up to the tail bud with the cloaca, and the somites were observed to settle down at this stage, and the curvature was decreased (Figure 1).

The embryos in the therapeutic dose group appeared more curved than those in the control group. Further, the vesicles were occluded, and the retinal pigmentation was reduced (Figure 2).

The vesicles in the supratherapeutic dose group appeared more distinct but smaller than those in the control and therapeutic dose groups. Further, the heart was clearly visible at its precise site, but its size was reduced, and the somites were apparently evident. However, the supratherapeutic dose group exhibited no morphological findings related to neural tube defects in the macroscopic examination (Figure 2).

After 72 hours of incubation, in the microscopic examination of the embryos using hematoxylin \& eosin staining, the continuity of the surface ectoderm was preserved; the neural tube and the notochord were at the correct site; and no mortalities, developmental delays, or neural tube defects were observed. Furthermore, the dorsal aorta and hind gut were detected, and the chorion and amnion surrounded the embryo (Figure $3 A-C$ ).

Immunohistochemical staining for mammalian target of rapamycin (mTOR), c-Jun N-terminal kinase (JNK), and microtubule-associated proteins 1A/1B light chain 3 (LC3) was performed in all the groups (Table I, Figure 4). mTOR staining was moderate to strong $(++/+++)$ in the control group, with staining intensity increasing toward the periphery. 

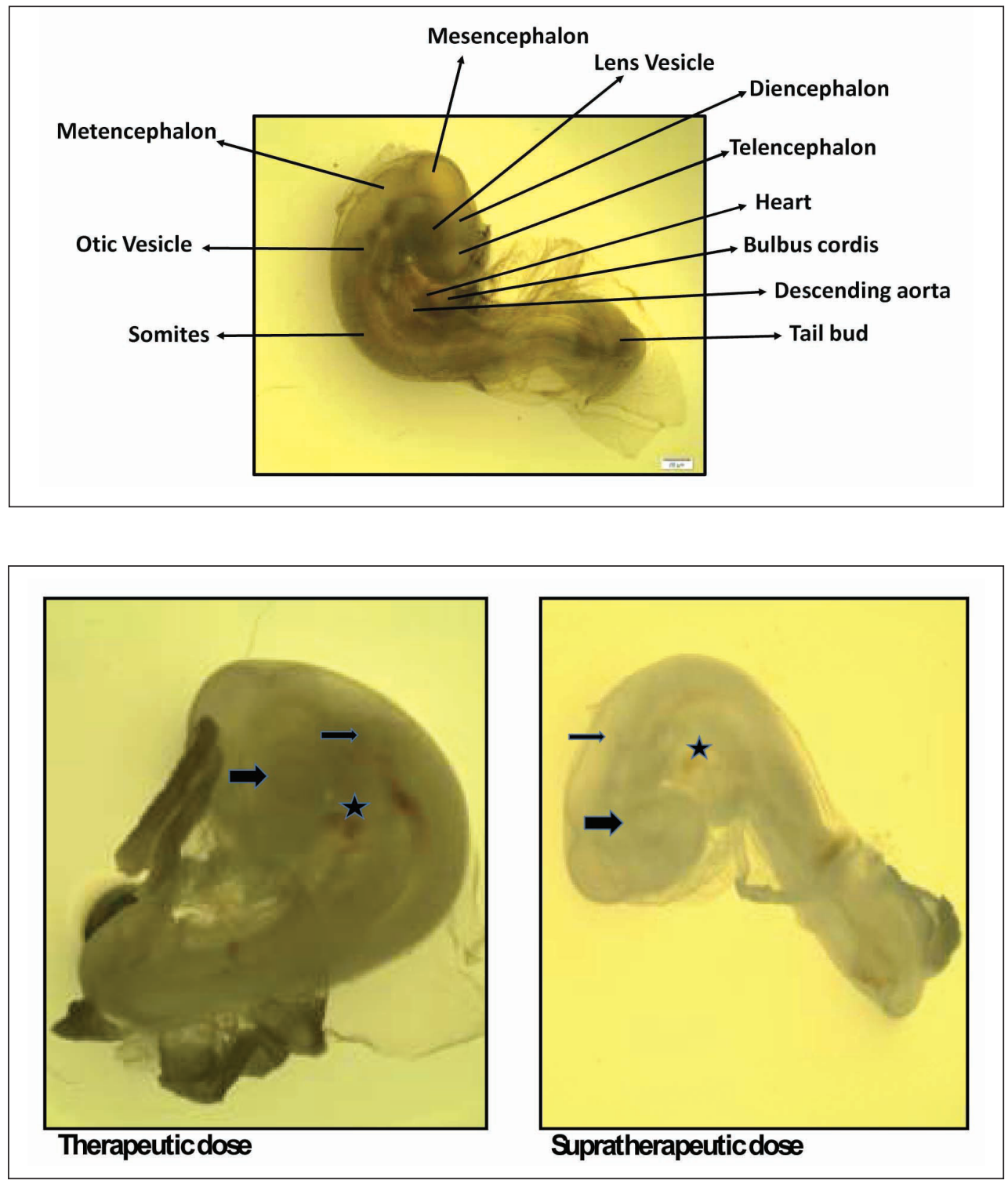

Figure 1: Macroscopic appearances of the control group embryos at 72 hours.

Figure 2: Macroscopic appearances of the therapeutic and supra therapeutic dose group embryos at 72 hours (optical magnification, $\times 200$ ). Otic vesicle (thin arrow); otic vesicle (thick arrow); heart (star).
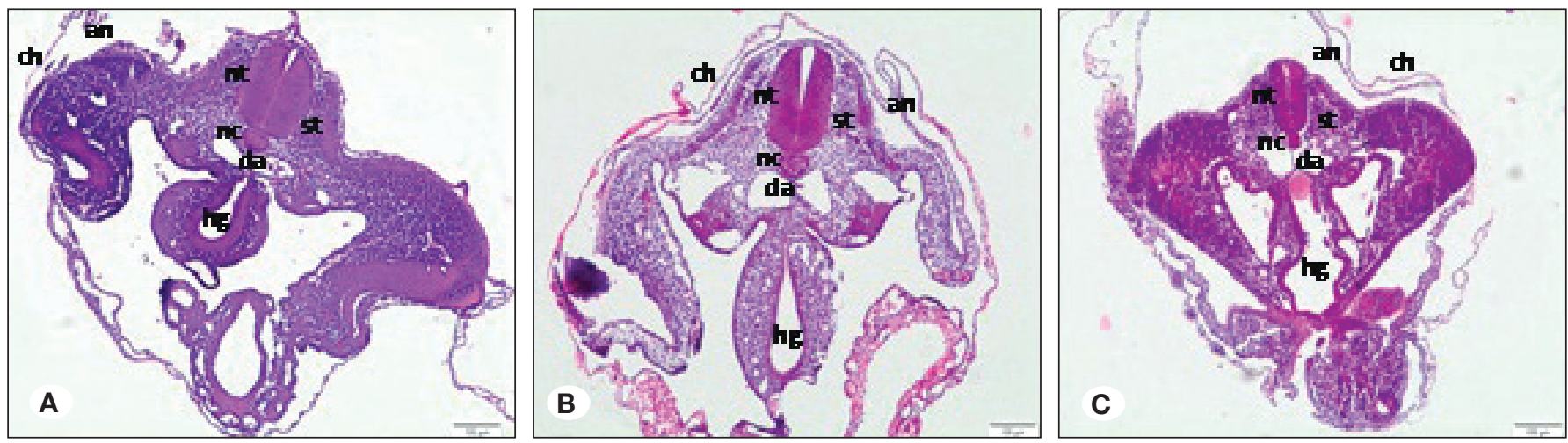

Figure 3: Appearances of hematoxylin and eosin-stained sections of control (A), therapeutic dose (B), and supratherapeutic dose (C) group embryos at 72 hours (optical magnification, $\times 200$ ). Chorion (ch), amnion (an), neural tube (nt), notochord (nc), somite (st), dorsal aorta (da), hind gut (hg). 
However, in the therapeutic and supratherapeutic dose groups, the staining intensity decreased $(+/++)$. Further, weak $(+)$ immunoreactivities of JNK were detected in the control and therapeutic dose groups, while moderate immunoreactivity was detected in the supratherapeutic dose group. In the control and therapeutic dose groups, LC3 staining was moderate $(++)$, but the staining area was larger. Furthermore, similar staining patterns in the neural tube, notochord, and other tissues were observed in the therapeutic dose and control groups. However, in the supratherapeutic dose group, the staining was strong $(+++)$ at every site, including the neural tube and the notochord, compared with that in the control and therapeutic dose groups.

Table I: Results of Immunohistochemical Staining

\begin{tabular}{|c|c|c|c|}
\hline & mTOR & JNK & LC3 \\
\hline Control group & $++/+++$ & + & ++ \\
\hline Therapeutic dose group & $+/++$ & + & ++ \\
\hline $\begin{array}{l}\text { Supratherapeutic dose } \\
\text { group }\end{array}$ & $+/++$ & ++ & +++ \\
\hline
\end{tabular}

+: weak expression, ++: moderate expression, +++: strong expression. mTOR: Mammalian target of rapamycin, JNK: c-Jun N-terminal kinase, LC3: microtubule-associated proteins 1A/1B light chain 3.

\section{DISCUSSION}

Neural tube development includes several processes, such as the development of the neural plate and the augmentation of the lateral neural folds, which ultimately fuse to construct the neural tube (7). The disruption of any of these processes causes neural tube defects, primarily spina bifida. The chick embryo was used in this study because it serves as an exceptional model for the investigation of prenatal fetus development, including neural tube closure. Moreover, several earlier reports have employed this model to examine the influence of different compounds, such as cotinine, meloxicam, and phenytoin, on early embryonic neural tube development $(5,8,11,13,34,35)$.

The majority of anticonvulsant drugs, such as phenytoin, carbamazepine, valproate, and phenobarbital, have been correlated to fetal abnormalities, including growth and developmental delay, microcephaly, orofacial clefts, digital anomalies, and neural tube defects (15). The possible mechanism underlying the induction of embryonic malformations by all these drugs has been reported to be mediated through apoptosis (2). Among these drugs, carbamazepine was considered to exert the fewest teratogenic effects until recently developed anticonvulsant drugs, such as pregabalin and gabapentin, were introduced (15). However, data regarding the effects of such recently developed AEDs on the prenatal fetus remain scarce.

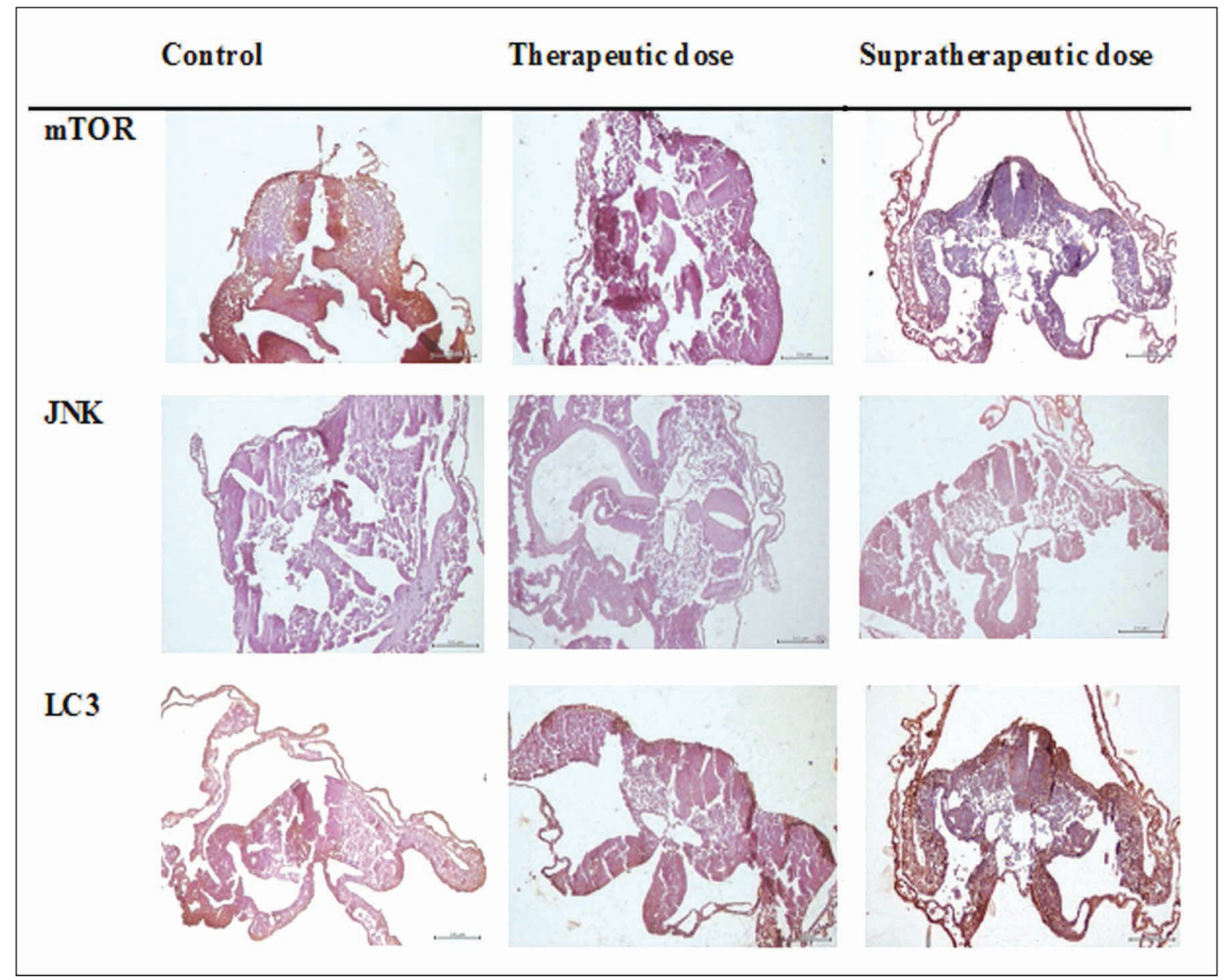

Figure 4: Immunohistochemical staining of the embryonic sections at 72 hours. 
The chemical structure of pregabalin is very similar to that of gabapentin, yet pregabalin is more efficacious and is more rapidly absorbed. Recent studies have reported that pregabalin decreases the release of central neurotransmitters by binding to the a2 $\delta-1$ subunit of the voltage-dependent $\mathrm{Ca}^{+2}$ channel, virtually inactivating the GABA receptors. AEDs particularly affect brain development; however, in the case of pregabalin, limb deformities have been reported to be the most commonly observed disorders (22). In the present study, the administration of therapeutic and supratherapeutic doses of pregabalin resulted in the formation of curved embryos compared with the normal embryos in the control group. Further, the vesicles were occluded and the retinal pigmentation was reduced following pregabalin administration. These results may be caused by increased apoptosis (a type I programmed cell death) involving the activation of the caspase- 3 protease pathway. The involvement of caspases in the fetal abnormalities has been previously demonstrated with other teratogens (38).

Apoptosis plays a crucial role in the origin, development, and equilibrium of the developing central nervous system (CNS) by contributing to the folding and fusion of the neural walls. Previous studies have reported that neural tube defects are associated with excessive neuroepithelial apoptosis $(14,31)$. Further, apoptosis is primarily regulated by the $\mathrm{Bcl}-2$ protein family, adaptor protein Apaf1, and cysteine protease caspase family. In addition, DNA methylation plays a significant role during early embryogenesis, and impaired methylation disrupts neurulation $(28,30,36)$. Furthermore, neurogenesis involves the down regulation of pluripotent genes (Oct4, Nanog, and Rex1) and the anti-neural REST/Scp1 during CNS development $(21,37)$, the expressions or mutations of which affect neural tube closure. Moreover, a recent study has reported that MARCKS, an F-actin-binding protein, is crucial during gastrulation and neurulation for maintaining neuroepithelial polarity. The phosphorylation of MARCKS by PKC leads to the impairment of the cell polarity of the chick neural plate, ultimately hampering neurulation (1).

In another study, pregabalin did not exhibit any teratogenic effects following its administration to rats at doses up to and including $2500 \mathrm{mg} / \mathrm{kg} /$ day (25). However, in the present study, pregabalin exerted teratogenic effects at much lower doses of 600 and $1200 \mathrm{mg} / 70 \mathrm{~kg}$.

Reportedly, the mTOR signaling pathway regulates autophagy (a type II programmed cell death) by inhibiting its initiation (16). In the present study, mTOR expression was moderate to strong in the control group, indicating a decrease in apoptosis rate, whereas mTOR expression decreased in the therapeutic and supratherapeutic dose groups, indicating an increase in apoptosis rate through enhanced autophagy following pregabalin administration to digest the cellular components and dysfunctional proteins that are no longer useful.

JNK plays a vital role in the initiation of both extrinsic and mitochondrial intrinsic apoptotic pathways. It activates apoptotic signaling by upregulating pro-apoptotic genes through the transactivation of specific transcription factors or by directly regulating the activities of mitochondrial pro- and anti-apoptotic proteins through distinct phosphorylation events (9). In the present study, weak JNK expression was observed in the control and therapeutic dose groups, whereas moderate expression was detected in the supratherapeutic dose group, indicating the activation of the apoptotic pathway.

It is well known that autophagy is regulated by a series of Atg genes. During the regulation of autophagy, Atg8 (LC3) is specifically cleaved and lapidated to become LC3-II, which is deployed to the autophagosome membrane. Increased levels of LC3-II proteins and LC3-II-containing autophagosomes are the key biomarkers for autophagy (17). LC3 staining in the current study was moderate in the control and therapeutic dose groups and strong in the supratherapeutic dose group, indicating increased autophagy in the supratherapeutic group following pregabalin administration.

\section{- CONCLUSION}

Pregabalin administration induced neural tube defects and fetal malformations in the chick embryo most probably through increased autophagy due to enhanced apoptosis in the prenatal fetus. This study provides an insight into the effects of pregabalin on the recently developed AED's on embryonic neural tube development, which should be explored through further research.

\section{- REFERENCES}

1. Aparicio G, Arruti C, Zolessi FR: MARCKS phosphorylation by PKC strongly impairs cell polarity in the chick neural plate. Genesis 56: e23104, 2018

2. Bittigau P, Sifringer M, Genz K, Reith E, Pospischil D, Govindarajalu S, Dzietko M, Pesditschek S, Mai I, Dikranian $\mathrm{K}$, Olney JW, Ikonomidou C: Antiepileptic drugs and apoptoticneuro degeneration in the developing brain. Proc Natl Acad Sci USA 99: 15089-15094, 2002

3. Bulau P, Paar WD, von Unruh GE: Pharmacokinetics of oxcarbazepine and 10- hydroxycarbazepine in the newborn child of an oxcarbazepine-treated mother. Eur $\mathrm{J}$ Clin Pharmacol 34: 311-313, 1988

4. Caglayan S, Kayhan B, Mentesoglu S, Aksit S: Changing incidence of neural tube defects in Aegean Turkey. Paediatr Perinat Epidemol 3: 62-65, 1989

5. Cetinkal A, Colak A, Topuz K, Demircan MN, Simsek H, Berber U, Umur AS, Selcuki M, Vatansever HS: The effects of meloxicam on neural tube development in the early stage of chick embryos. Turkish Neurosurg 20: 111-116, 2010

6. Chernoff N, Rogers EH, Gage MI, Francis BM: The relationship of maternal and fetal toxicity in developmental toxicology bioassays with notes on the biological significance of the "no observed adverse effect level”. Reprod Toxicol 25: 192-202, 2008

7. Copp AJ, Brook FA, Estibeiro JP, Shum AS, Cockroft DL: The embryonic development of mammalian neural tube defects. Prog Neurobiol 35: 363-403, 1990

8. Dalgic A, Armagan E, Helvacioglu F, Okay O, Daglioglu E, Take G, Unlu A, Belen D: High dose cotinine may induce neural tube defects in a chick embryo model. Turkish Neurosurg 19: 224-229, 2009 
9. Dhanasekaran DN, Reddy EP: JNK signaling in apoptosis. Oncogene 27: 6245-6251, 2008

10. Etemad L, Afshar M, Mohammadpour AH, Vahdati Mashhadi N, Moallem SA: Teratogenic effects of pregabalin in mice. Iran J Basic Med Sci 16: 1065-1070, 2013

11. Guney O, Canbilen A, Konak A, Acar O: The effects of folic acid in the prevention of neural tube development defects caused by phenytoin in early chick embryos. Spine 28: 442445, 2003

12. Hamburger V, Hamilton HL: A series of normal stages in the development of the chick embryo. J Morphol 88: 49-92, 1951

13. Hamilton JW, Kaltreider RC, Bajenova OV, Ihnat MA, McCaffrey J, Turpie BW, Rowell EE, Oh J, Nemeth MJ, Pesce CA, Lariviere JP: Molecular basis for effects of carcinogenic heavy metals on inducible gene expression. Environ Health Perspect 106: 1005-1015, 1998

14. Harris MJ, Juriloff DM: Mouse mutants with neural tube closure defects and their role in understanding human neural tube defects. Birth Defects Res A Clin Mol Teratol 79:187-210, 2007

15. Isoherranen N, Spiegelstein O, Roeder M, Triplett AA, Schurig V, Finnel RH: Developmental outcome of levetiracetam, its major metabolite in humans, 2-pyrrlidinone $\mathrm{N}$-butyric acid, and itsenantiomer (R)-alpha-ethyl-oxo-pyrolidineacetamide in a mouse model of teratogenicity. Epilepsia 44: 1280-1288, 2003

16. Jaboin JJ, Shinohara ET, Moretti L, Yang ES, Kaminski JM, Lu B: The role of mTOR inhibition in augmenting radiation induced autophagy. Technol Cancer Res Treat 6:443-447, 2007

17. Kabeya Y, Mizushima N, Ueno T, Yamamoto A, Kirisako T, Noda T, Kominami E, Ohsumi Y, Yoshimori T: LC3, a mammalian homologue of yeast Apg8p, is localized in autophagosome membranes after processing. Embo J 19: 5720-5728, 2000

18. Kawada K, Itop S, Kusaka T, Isobe K, Ishii M: Pharmacokinetics of zonisamide in perinatal period. Brain Dev 24: 95-97, 2002

19. Kim J, Kondratyev A, Gale K: Antiepileptic drug-induced neuronal cell death in the immature brain: Effects of carbamazepine, topiramate and levetiracetam as monotheraphy versus polytheraphy. J Pharmacol Exp Ther 323: 165-173, 2007

20. Kondo T, Kaneko S, Amano Y, Egewa I: Preliminary report on teratogenic effects of zonisamide in the offspring of treated women with epilepsy. Epilepsia 37: 1242-1244, 1996

21. Li D, Lu C, Wang J, Hu W, Cao Z, Sun D, Xia H, Ma X: Developmental mechanisms of arsenite toxicity in zebrafish (danio rerio) embryos. Aquat Toxicol 91: 229-237, 2009

22. Lingamaneni $\mathrm{R}$, Hemmings $\mathrm{HJ}$ : Differential interaction of anaesthetics and antiepileptic drugs with neuronal $\mathrm{Na}^{+}$ channels, $\mathrm{Ca}^{2+}$ channels, and $\mathrm{GABA}(\mathrm{A})$ receptors. $\mathrm{Br} \mathrm{J}$ Anaesth 90: 199-211, 2003

23. Meador KJ, Penovich P, Baker GA, Pennel PB, Bromfield E, Pack A, Liporace JD, Sam M, Kalayjian LA, Thurman DJ, Moore E, Loring DW: Antiepileptic drug use in women of childbearing age. Epilepsy Behav 15: 339-343, 2009
24. Morrow J, Russell A, Guthrie E, Parsons L, Robertson I, Waddell R, Irwin B, Mc Givern RC, Morrison PJ, Craig J: Malformation risks of antiepileptic drugs in pregnancy: $A$ prospective study from the UK Epilepsy and Pregnancy Register. J Neurol Neurosurg Psychiatry 77: 193-198, 2006

25. Morse DC, Henck JW, Bailey SA: Developmental toxicity studies with pregabalin in rats: Significance of alterations in skull bone morphology. Birth Defects Res Part B 107: 94-107, 2006

26. Mountouris G: Safety of the newer antiepileptic drug oxcarbazepine during pregnancy. Curr Med Res Opin 21: 693701, 2005

27. Myllynen P, Pienimaki P, Jouppila P, Vahakangas K: Transplacental passage of oxcarbazepine and its metabolites in vivo. Epilepsia 42: 1482-1485, 2001

28. Oda $M$, Okano $M$ : DNA methylation during mouse early embryo genesis. Tanpakushitsu Kakusan Koso 51:2027-2034, 2006

29. Ohman I, Vitols S, Luef G, Soderfeldt B, Tomson T: Topiramate kinetics during delivery, lactation and in the neonate: Preliminary observations. Epilepsia 43: 1157-1160, 2002

30. Park JG, Chapman VM: DNA methylation patterns and sequence transitions of the cpg Island of mouse hprt during early embryogenesis. Mol Cells 7: 259-263, 1997

31. Phelan SA, Ito M, Loeken MR: Neural tube defects in embryos of diabetic mice: Role of the pax-3 gene and apoptosis. Diabetes 46: 1189-1197, 1997

32. Rabinowicz A, Meischenguiser R, Ferraso SM, D' Giano $\mathrm{CH}$, Carrazana EJ: Single center, 7-year experience of oxcarbazepine of oxcarbazepine exposure during pregnancy. Epilepsia 43: 2008-2009, 2002

33. Stahl SM, Porreca F, Taylor CP, Cheung R, Thorpe AJ, Clair A: The diverse therapeutic actions of pregabalin: Is a single mechanism responsible for several pharmacological activities? Trends Pharmacol Sci 34: 332-339, 2013

34. Temiz C, Temiz P, Demirel A, Sayın M, Umur AŞ, Ozer FD: Effect of sodium phenytoin concentration on neural tube development in the early stages of chick embryo development. J Clin Neurosci 16: 307-311, 2009

35. Tufan AC, Akdogan I, Adiguzel E: Shell-less culture of the chick embryo as a model system in the study of developmental neurobiology. Neuroanatomy 3: 8-11, 2004

36. Van der Linden IJ, Heil SG, van Egmont Petersen M, van Straaten HW, denHeijer M, Blom HJ: Inhibition of methylation and changes in gene expression in relation to neural tube defects. Birth Defects Res A Clin Mol Teratol 82:676-683, 2008

37. Visvanathan J, Lee S, Lee B, Lee JW, Lee SK: The microrna mir-124 antagonizes the anti-neural rest/scp1 pathway during embryonic CNS development. Genes Dev 21: 744-749, 2007

38. Umur AS, Selcuki M, Bursali A, Umur N, Kara B, Vatansever HS, Duransoy YK: Simultaneous folate intake may prevent adverse effect of valproic acid on neurulating nervous system. Childs Nerv Syst 28: 729-737, 2012 